\title{
Is metabolic syndrome related with coronary artery disease severity and complexity: An observational study about IDF and AHA/NHLBI metabolic syndrome definitions
}

\author{
Ahmet Çağrı Aykan, İlker Gül1 ${ }^{1}$ Ezgi Kalaycıoğlu', Tayyar Gökdeniz ${ }^{1}$, \\ Engin Hatem ${ }^{1}$, Ümit Menteşe $e^{2}$, Banu Şahin Y1ldız ${ }^{3}$, Mustafa Yıldız \\ ${ }^{1}$ Department of Cardiology, Ahi Evren Chest Cardiovascular Surgery \\ Education and Research Hospital, Trabzon, Turkey \\ ${ }^{2}$ Department of Cardiovascular Surgery, Ahi Evren Chest Cardiovascular Surgery \\ Education and Research Hospital, Trabzon, Turkey
}

${ }^{3}$ Department of Medicine, Kartal Lutfi Kırdar Education and Research Hospital, Istanbul, Turkey

${ }^{4}$ Department of Cardiology, Istanbul University Haseki Cardiology Institute, Istanbul, Turkey

\begin{abstract}
Background: The aim of the present study was to assess the relation between metabolic syndrome (MS) and coronary artery disease (CAD) complexity, assessed by Syntax score (SS), and severity in non-diabetic patients with stable CAD who underwent coronary angiography, and to evaluate whether the MS defined by different definitions, including International Diabetes Federation (IDF) and American Heart Association/National Heart Lung Blood Institute (AHA/NHLBI) guidelines, similarly correlated with SS.
\end{abstract}

Methods: The present study is cross sectional and observational with prospective inclusion of 248 consecutive patients (157 male) who underwent coronary angiography due to stable CAD. Results: The prevalence of MS was 54.4\% according to IDF definition and $50.4 \%$ according to AHA/NHLBI definition. MS score according to IDF definitions $(r=0.446, p<0.001), M S$ score according to AHA/NHLBI definitions ( $r=0.341, p<0.001$ ) were moderately correlated with SS. In Fisher $r$ to $z$ transformation test the correlations of the presence of MS according to IDF and AHA/NHLBI definitions with SS were not statistically significant ( $p=0.168, z=-1.38)$. The systolic blood pressure ( $p<0.001, B=0.354,95 \% C I=-0.308$ to 0.228$)$, diastolic blood pressure ( $p=0.006, B=-0.194,95 \% C I=-0.333$ to -0.056$)$, age $(p=0.014, B=0.147$, $95 \% C I=0.029$ to 0.264), left ventricular ejection fraction $(p=0.031, B=-0.150,95 \% C I$ $=-0.286$ to -0.014$)$, waist/hip circumference $(p<0.001, B=45.713,95 \% C I=23.235$ to $68.1919)$ and $\log 10$ high density lipoprotein $(p<0.001, B=-22.209,95 \% C I=-33.298$ to $-11.119)$ were the independent predictors of SS in linear regression analysis.

Conclusions: $M S$ is associated with the presence and complexity of $C A D$. Besides the presence of discrepancy in the limits of waist circumference, both IDF and AHA/NHLBI criteria were similarly correlated with CAD complexity. (Cardiol J 2014; 21, 3: 245-251)

Key words: metabolic syndrome, Syntax score, coronary, cardiovascular, IDF, AHA/NHLBI

Address for correspondence: Dr Ahmet Çağrı Aykan, Department of Cardiology, Ahi Evren Chest and Cardiovascular Surgery Education and Research Hospital, Soğuksu Mahallesi, Çamlık Caddesi, 61040 Trabzon, Turkey, tel: 905058689461, fax: 904622310483, e-mail: ahmetaykan@yahoo.com

Received: 11.05.2013 Accepted: 27.08.2013 


\section{Introduction}

The metabolic syndrome (MS) is characterized by visceral adiposity, insulin resistance, dyslipidemia and increased blood pressure [1,2]. The American Heart Association/National Heart Lung Blood Institute (AHA/NHLBI) and International Diabetes Federation (IDF) criteria are widely used for defining MS. Patients with MS are at increased risk of cardiovascular events (2-fold increase in cardiovascular outcomes, cardiovascular mortality, myocardial infarction and stroke, and a 1.5 -fold increase in all-cause mortality) [3, 4]. However, there is still a controversy about the association of MS with stable coronary artery disease (CAD) [5-10].

The Syntax score (SS) is a widely accepted CAD complexity score based on lesion morphology. The SS was correlated with cardiovascular mortality and morbidity [11]. Higher SS was correlated with poor outcome and major adverse cardiovascular and cerebrovascular events (MACCE) in many studies [11-14]. Recently it has been reported that atherosclerotic plaque compositions of patients with MS who did not have diabetes mellitus had similar features as patients with diabetes $[15,16]$. The CAD complexity is not adequately evaluated in patients with MS. The aim of the present study was to assess the relationship between MS and CAD complexity and severity in non-diabetic patients with stable CAD who underwent coronary angiography (CAG) and to evaluate whether the MS defined by different definitions, including IDF and AHA/NHLBI guidelines, similarly correlated with SS.

\section{Methods}

The present study is cross sectional and observational with prospective inclusion of 248 consecutive patients ( 157 male) who underwent CAG due to stable CAD (stable angina and/or ischemia) between May 2012 and February 2013. Informed consent was obtained from all the subjects, and the investigation conforms to the principles outlined in the Declaration of Helsinki. The study protocol was approved by the ethics committee.

Patients with acute coronary syndrome (ACS) and previous myocardial infarction, history of myocardial revascularization procedure, congenital heart disease, dilated or constrictive cardiomyopathy, diabetes mellitus, peripheral arterial disease, moderate to severe valvular heart disease, cerebrovascular disease, congestive heart failure (left ventricular ejection fraction $[\mathrm{LVEF}] \leq 40 \%$ ), renal failure (estimated creatinine clearance $<60 \mathrm{~mL} / \mathrm{min} / 1.73 \mathrm{~m}^{2}$ ) and cancer were excluded from the study.

The MS was defined by the most recent definition from AHA/NHLBI and IDF consisting of $\geq 3$ of the following criteria: fasting glucose $\geq 100 \mathrm{mg} / \mathrm{dL}$ or antidiabetic treatment; blood pressure $\geq 130 / 85 \mathrm{~mm} \mathrm{Hg}$ or antihypertensive medication; triglycerides $\geq 150 \mathrm{mg} / \mathrm{dL}$ or specific treatment for this lipid abnormality; high density lipoprotein cholesterol (HDL-C) $<50 \mathrm{mg} / \mathrm{dL}$ in women and $<40 \mathrm{mg} / \mathrm{dL}$ in men or specific treatment for this lipid abnormality; waist circumference (WC) $\geq 88 \mathrm{~cm}$ in women for AHA/NHLBI and $\geq 80$ for IDF and $\geq 102 \mathrm{~cm}$ in men for AHA/NHLBI and $\geq 94$ for IDF $[1,2]$.

Weights of the patients, in light clothes and without shoes, were measured in kilograms, and their heights were also measured. Body mass index (BMI) was calculated by dividing body weight in kilograms by the square of body height in meters. WC was measured between the last rib and iliac crest on the midline while the patient was standing. Hip circumference ( $\mathrm{HC}$ ) was measured by using the line between the right and left great trochanter of the femur. Transthoracic echocardiographic assessment (Vivid S5 General Electric, Norway) was performed in patients according to the standards of the American Society of Echocardiography. Left ventricular mass (LVM) was calculated according to Devereux formula:

$$
\begin{gathered}
\text { LVM: } 0.8 \times 1.04 \times \\
\times\left[(\text { LVEDD }+ \text { IVS }+\mathrm{PW})^{3}-\mathrm{LVEDD}^{3}\right]+0.6 \\
\text { LVM index }(\mathrm{LVMI}): \mathrm{LVM} / \text { body surface area }
\end{gathered}
$$

Blood pressure was measured, in compliance with World Health Organization guidelines, by using a mercury sphygmomanometer (ERKA, Germany) with a cuff appropriate to the arm circumference, in patients at rest for $20 \mathrm{~min}$ (Korotkoff phase I for systolic blood pressure [SBP] and $\mathrm{V}$ for diastolic blood pressure [DBP]) at least 3 times and the average of the measurements was calculated. Hypertension was defined by a previous diagnosis of hypertension or the presence of $\mathrm{SBP} \geq 140 \mathrm{~mm} \mathrm{Hg}$ or $\mathrm{DBP} \geq 90 \mathrm{~mm} \mathrm{Hg}$ (mean of 2 consecutive measurements). Diabetes was defines as fasting plasma glucose $\geq 126 \mathrm{mg} / \mathrm{dL}$ or plasma glucose level $\geq 200 \mathrm{mg} / \mathrm{dL} 2 \mathrm{~h}$ after the $75 \mathrm{mg}$ oral glucose tolerance test or symptoms of hyperglycemia accompanied by casual plasma glucose $\geq 200 \mathrm{mg} / \mathrm{dL}$ or $\mathrm{HbA} 1 \mathrm{c} \geq 6.5 \%$ or patients using antidiabetic medications. 


\section{Pulse pressure $=\mathrm{SBP}-\mathrm{DBP}$ \\ Mean blood pressure $=\mathrm{SBP}+2 \times \mathrm{DBP} / 3$}

All cases underwent diagnostic coronary angiography according to Judkins technique. The left main, left anterior descending, circumflex, and right coronary arteries were defined as major coronary vessels. Patients with slow flow in the absence of any discernible lesions were also excluded from the study. Each coronary lesion producing $\geq 50 \%$ luminal obstruction in vessels with a diameter $\geq 1.5 \mathrm{~mm}$ was separately scored and added to provide the vessel SS, and then summed to provide the overall patient SS (www.Syntaxscore.com). The SS was calculated using dedicated software (version 2.11) that integrates the number of lesions with their specific weighting factors based on the amount of myocardium distal to the lesion and the morphologic features of each single lesion. Obstructive CAD was defined as stenosis of 50\% or more in any coronary vessel. Coronary arteries without luminal irregularity on CAG were accepted normal coronary arteries.

Patients who self-reported as having smoked during the previous 6 months were classified as smokers. Venous blood samples were drawn after a 12 -h overnight fast. Serum glucose, total cholesterol and triglycerides were determined using standard automatic enzymatic methods. HDL-C was determined after specific precipitation and low density lipoprotein cholesterol (LDL-C) was determined by the Friedewald formula.

\section{Statistical analysis}

Analyses were conducted using SPSS 17.0 (SPSS for Windows 17.0, Chicago, Illinois). Continuous variables were expressed as mean \pm standard deviation (for parameters with normal distribution), median (interquartile range, IQR) (for parameters with none normal distribution) and categorical variables were expressed as percentages. Comparison of categorical variables between the groups was performed using the $\chi^{2}$ test. Analysis of normality was performed with the KolmogorovSmirnov test. Comparisons of continuous variables between the two groups were performed using the independent samples t-test or Mann-Whitney U test, as appropriate. Pearson and Spearman correlation analyses were used to analyze correlates of SS. To compare the correlation coefficients of the presence of MS according to IDF and AHA/ /NHLBI definitions with SS, Fisher $r$ to $z$ transformation was performed. Multivariate regression analysis was performed to find the independent predictors of SS. The logarithmic transformation was performed to none normally distributed variables including HDL, fasting glucose and triglyceride levels. The WC, SBP, DBP, sex, age, BMI, creatinine, hypertension, hyperlipidemia, LVEF, $\mathrm{WC} / \mathrm{HC}$ ratio, log 10 fasting blood glucose, $\log 10$ triglyceride, $\log 10 \mathrm{HDL}$ values were entered to linear regression analysis. A 2 -sided $\mathrm{p}<0.05$ was considered significant within $95 \%$ confidence interval (CI).

\section{Results}

A total of 248 patients, $63.3 \%$ male were included in the study, with a mean age of $60.95 \pm$ \pm 9.42 (range: $36-83$ ) years. Baseline patients' characteristics are shown in Table 1 . Significant CAD was present in 133 (53.6\%) patients, but significantly more common among men $(71.48 \%$ vs. $28.6 \%, p=0.004)$. The SS was also significantly higher in men: $10.03 \pm 11.10$ vs. $5.62 \pm 8.62, \mathrm{p}=$ $=0.001$. Patients with significant $\mathrm{CAD}$ were older and more commonly male. Hypertension and hyperlipidemia were common among patients with significant $\mathrm{CAD}$. Patients with significant $\mathrm{CAD}$ had higher BMI, waist circumference, $\mathrm{WC} / \mathrm{HC}$ ratio, fasting blood glucose, pulse and mean arterial pressure. MS was common among patients with significant CAD.

The prevalence of MS was $54.4 \%$ according to IDF definitions and $50.4 \%$ according to AHA/ /NHLBI definition. Patients' characteristics according to IDF and AHA/NHLBI definitions are shown in Table 2.

The SS was weakly correlated with age, sex, hypertension, BMI, creatinine, mean arterial pressure and LVEF in correlation analysis. The WC $(\mathrm{r}=0.299, \mathrm{p}<0.001), \mathrm{WC} / \mathrm{HC}$ ratio $(\mathrm{r}=0.413$, $\mathrm{p}<0.001)$, HDL $(\mathrm{r}=-0.283, \mathrm{p}<0.001)$, pulse pressure $(\mathrm{r}=0.334, \mathrm{p}=0.001)$, MS score according to IDF definitions ( $r=0.360, p<0.001)$, MS score according to AHA/NHLBI definitions ( $\mathrm{r}=0.361$, $\mathrm{p}<0.001)$, presence of MS score according to IDF definitions ( $r=0.446, \mathrm{p}<0.001$ ), MS score according to AHA/NHLBI definitions ( $r=0.341$, $\mathrm{p}<0.001$ ) were moderately correlated with SS (Table 3). In Fisher $r$ to $z$ transformation test the correlations of the presence of MS according to IDF and AHA/NHLBI definitions with SS was not statistically significant $(\mathrm{p}=0.168, \mathrm{z}=-1.38)$.

The SBP $(\mathrm{p}<0.001, \mathrm{~B}=0.354,95 \% \mathrm{CI}=-0.308$ to 0.228$), \mathrm{DBP}(\mathrm{p}=0.006, \mathrm{~B}=-0.194,95 \%$ $\mathrm{CI}=-0.333$ to -0.056$)$, age $(\mathrm{p}=0.014, \mathrm{~B}=0.147$, $95 \% \mathrm{CI}=0.029$ to 0.264$), \operatorname{LVEF}(\mathrm{p}=0.031$, 
Table 1. Baseline patients' characteristics.

\begin{tabular}{|c|c|c|c|}
\hline Variable & Significant CAD (n = 133) & No CAD ( $n=115)$ & $\mathbf{P}$ \\
\hline Age [year] & $62.28 \pm 9.45$ & $59.41 \pm 9.18$ & 0.016 \\
\hline Male & $95(71.4 \%)$ & $62(53.9 \%)$ & 0.004 \\
\hline Hypertension & $81(60.9 \%)$ & $51(44.3 \%)$ & 0.009 \\
\hline Hyperlipidemia & $52(42.9 \%)$ & $27(23.5 \%)$ & 0.001 \\
\hline Smoking & $51(38.3 \%)$ & $44(38.3 \%)$ & 0.989 \\
\hline Body mass index $\left[\mathrm{kg} / \mathrm{m}^{2}\right]$ & $28.48 \pm 3.46$ & $26.80 \pm 4.97$ & 0.003 \\
\hline $\mathrm{WC}[\mathrm{cm}]$ & $93.86 \pm 11.15$ & $83.75 \pm 15.64$ & $<0.001$ \\
\hline $\mathrm{HC}[\mathrm{cm}]$ & $97.76 \pm 7.35$ & $97.11 \pm 8.03$ & 0.509 \\
\hline WC/HC ratio & $0.96 \pm 0.09$ & $0.86 \pm 0.11$ & $<0.001$ \\
\hline Triglyceride [mg/dL] & $128(82)$ & $153(119)$ & 0.011 \\
\hline High density lipoprotein [mg/dL] & $39(12.5)$ & $48(15)$ & 0.001 \\
\hline Glucose [mg/dL] & $102(18.5)$ & $98(12)$ & 0.001 \\
\hline Low density lipoprotein [mg/dL] & $143.91 \pm 42.68$ & $142.60 \pm 33.89$ & 0.787 \\
\hline Total cholesterol [mg/dL] & $205.84 \pm 49.42$ & $211.34 \pm 36.09$ & 0.314 \\
\hline Creatinine $[\mathrm{mg} / \mathrm{dL}]$ & $0.86 \pm 0.21$ & $0.82 \pm 0.19$ & 0.092 \\
\hline eGFR $\left[\mathrm{mL} / \mathrm{min} / 1.73 \mathrm{~m}^{2}\right]$ & $102.44 \pm 31.12$ & $100.20 \pm 35.50$ & 0.597 \\
\hline Systolic blood pressure [mm Hg] & $146.08 \pm 13.03$ & $137.94 \pm 16.23$ & $<0.001$ \\
\hline Diastolic blood pressure [mm Hg] & $87.43 \pm 9.18$ & $84.80 \pm 11.18$ & 0.046 \\
\hline Pulse pressure [mm Hg] & $58.65 \pm 11.15$ & $53.14 \pm 10.97$ & $<0.001$ \\
\hline Mean arterial pressure $[\mathrm{mm} \mathrm{Hg}]$ & $106.98 \pm 9.23$ & $102.51 \pm 12.01$ & 0.001 \\
\hline MS score IDF & $3.29 \pm 1.10$ & $2.03 \pm 1.35$ & $<0.001$ \\
\hline MS score AHA & $3.07 \pm 1.09$ & $1.91 \pm 1.21$ & $<0.001$ \\
\hline MS IDF & $101(71.9 \%)$ & $34(29.6 \%)$ & $<0.001$ \\
\hline MS AHA & $92(69.2 \%)$ & $33(28.7 \% 9$ & $<0.001$ \\
\hline Left ventricular ejection fraction [\%] & $62.24 \pm 8.14$ & $63.86 \pm 7.99$ & 0.116 \\
\hline Left ventricular mass index $\left[\mathrm{g} / \mathrm{m}^{2}\right]$ & $117.47 \pm 38.94$ & $116.84 \pm 37.95$ & 0.897 \\
\hline
\end{tabular}

CAD - coronary artery disease; WC - waist circumference; HC — hip circumference; eGFR - estimated glomerular filtration rate;

MS IDF — metabolic syndrome according to International Diabetes Federation definition; MS AHA/NHLBI — metabolic syndrome according to American Heart Association/National Heart Lung Blood Institute definition

$\mathrm{B}=-0.150,95 \% \mathrm{CI}=-0.286$ to -0.014$), \mathrm{WC} / \mathrm{HC}$ $(\mathrm{p}<0.001, \mathrm{~B}=45.713,95 \% \mathrm{CI}=23.235-68.1919)$ and $\log 10$ HDL $(\mathrm{p}<0.001, \mathrm{~B}=-22.209,95 \%$ $\mathrm{CI}=-33.298$ to -11.119$)$ were the independent predictors of SS in linear regression analysis (Table 4).

\section{Discussion}

There is limited data regarding the association between MS and the presence and complexity of CAD in non-diabetic patients. To our knowledge this is the first study evaluating and comparing the association of CAD complexity with MS defined by IDF and AHA/NHLBI in diabetic patients who underwent coronary angiography due to suspected stable CAD. We found that MS is associated with the significant angiographic $\mathrm{CAD}$ and presence of MS according to both AHA/NHLBI and IDF were similarly correlated with SS. Furthermore, there was no statistical difference for both definitions with the correlation of SS. The SBP, DBP, age, LVEF, WC/HC and log 10 HDL were the independent predictors of SS in linear regression analysis.

There are several definitions of MS $[1,2,17]$. Of these, the AHA/NHLBI and IDF definitions have emerged as the most widely used. Thus we used them. The main difference between these criteria concerns the measurement of abdominal obesity. Because of this discordance, IDF and AHA/NHLBI defined a consensus statement and they recommended the IDF cut points be used for non-Europeans and either the IDF or AHA/NHLBI cut points to be used for people of European origin until more data are available [18].

The MS was associated with increased cardiovascular disease, cardiovascular mortality, and stroke [4]. Patients with clinically manifest CAD have a high prevalence of MS. In patients hospitalized for ACS, the prevalence ranged between $43 \%$ and 
Table 2. The patients' features according to metabolic syndrome (MS) definitions.

\begin{tabular}{|c|c|c|c|c|c|c|}
\hline \multirow[t]{2}{*}{ Variable } & \multicolumn{2}{|c|}{ MS IDF } & \multirow[t]{2}{*}{$\mathbf{P}$} & \multicolumn{2}{|c|}{ MS AHA/NHLBI } & \multirow[t]{2}{*}{$\mathbf{P}$} \\
\hline & $\begin{array}{c}M S(+) \\
(n=135)\end{array}$ & $\begin{array}{c}\text { MS }(-) \\
(n=113)\end{array}$ & & $\begin{array}{c}M S(+) \\
(n=125)\end{array}$ & $\begin{array}{c}\text { MS(-) } \\
(n=123)\end{array}$ & \\
\hline Age [year] & $60.61 \pm 9.66$ & $61.35 \pm 9.15$ & 0.544 & $60.60 \pm 9.35$ & $61.30 \pm 9.51$ & 0.559 \\
\hline Male & $90(66.7 \%)$ & $67(59.3 \%)$ & 0.230 & $81(64.8 \%)$ & $76(61.8 \%)$ & 0.623 \\
\hline Hypertension & $87(64.4 \%)$ & $45(39.8 \%)$ & $<0.001$ & $80(64.0 \%)$ & $52(42.3 \%)$ & 0.001 \\
\hline Hyperlipidemia & $52(38.5 \%)$ & $32(28.3 \%)$ & 0.091 & $47(37.6 \%)$ & $37(30.1 \%)$ & 0.211 \\
\hline Smoking & $55(40.7 \%)$ & $40(35.4 \%)$ & 0.389 & $50(40 \%)$ & $45(36.6 \%)$ & 0.580 \\
\hline Body mass index $\left[\mathrm{kg} / \mathrm{m}^{2}\right]$ & $29.26 \pm 3.49$ & $25.84 \pm 4.45$ & $<0.001$ & $29.36 \pm 3.61$ & $26.02 \pm 4.31$ & $<0.001$ \\
\hline $\mathrm{WC}[\mathrm{cm}]$ & $95.76 \pm 12.11$ & $81.30 \pm 12.72$ & $<0.001$ & $95.94 \pm 12.52$ & $82.29 \pm 12.70$ & $<0.001$ \\
\hline $\mathrm{HC}[\mathrm{cm}]$ & $99.68 \pm 7.41$ & $94.80 \pm 7.11$ & $<0.001$ & $99.84 \pm 7.60$ & $95.04 \pm 6.96$ & $<0.001$ \\
\hline $\mathrm{WC} / \mathrm{HC}$ ratio & $0.96 \pm 0.10$ & $0.86 \pm 0.10$ & $<0.001$ & $0.96 \pm 0.11$ & $0.86 \pm 0.10$ & $<0.001$ \\
\hline Triglyceride [mg/dL] & $188(126)$ & $111(43)$ & $<0.001$ & $191(124)$ & $111(48)$ & $<0.001$ \\
\hline $\mathrm{HDL}[\mathrm{mg} / \mathrm{dL}]$ & $39(12)$ & $50(14.5)$ & $<0.001$ & $38(13)$ & $48(14)$ & $<0.001$ \\
\hline Glucose $[\mathrm{mg} / \mathrm{dL}]$ & $104(18)$ & $94.5(12.5)$ & $<0.001$ & 105 (19) & $95(13)$ & $<0.001$ \\
\hline LDL [mg/dL] & $149.03 \pm 39.71$ & $136.45 \pm 36.65$ & 0.011 & $149.63 \pm 40.17$ & $136.87 \pm 36.36$ & 0.009 \\
\hline Total cholesterol $[\mathrm{mg} / \mathrm{dL}]$ & $211.87 \pm 40.06$ & $204.23 \pm 40.63$ & 0.171 & $212.83 \pm 47.05$ & $203.88 \pm 39.80$ & 0.107 \\
\hline Creatinine $[\mathrm{mg} / \mathrm{dL}]$ & $0.84 \pm 0.20$ & $0.85 \pm 0.21$ & 0.648 & $0.83 \pm 0.20$ & $0.86 \pm 0.21$ & 0.421 \\
\hline eGFR, $\left[\mathrm{mL} / \mathrm{min} / 1.73 \mathrm{~m}^{2}\right]$ & $108.62 \pm 32.26$ & $92.79 \pm 32.32$ & $<0.001$ & $108.86 \pm 31.88$ & $93.82 \pm 32.87$ & $<0.001$ \\
\hline $\mathrm{SBP}[\mathrm{mm} \mathrm{Hg}]$ & $146.24 \pm 12.58$ & $137.60 \pm 16.57$ & $<0.001$ & $146.18 \pm 12.75$ & $138.36 \pm 16.34$ & $<0.001$ \\
\hline $\mathrm{DBP}[\mathrm{mm} \mathrm{Hg}]$ & $88.56 \pm 10.32$ & $83.39 \pm 9.39$ & $<0.001$ & $88.31 \pm 10.60$ & $84.07 \pm 9.39$ & 0.001 \\
\hline Pulse pressure [mm Hg] & $57.67 \pm 11.64$ & $54.20 \pm 10.82$ & 0.016 & $57.87 \pm 11.83$ & $54.28 \pm 10.66$ & 0.013 \\
\hline MAP [mm Hg] & $107.79 \pm 9.68$ & $101.47 \pm 11.14$ & $<0.001$ & $107.60 \pm 9.90$ & $102.17 \pm 11.07$ & $<0.001$ \\
\hline MS score & $3.79 \pm 0.76$ & $1.42 \pm 0.64$ & $<0.001$ & $3.61 \pm 0.73$ & $1.44 \pm 0.63$ & $<0.001$ \\
\hline LVEF [\%] & $63.48 \pm 8.80$ & $62.41 \pm 7.18$ & 0.299 & $64.06 \pm 7.64$ & $61.90 \pm 8.43$ & 0.035 \\
\hline LVMI $\left[\mathrm{g} / \mathrm{m}^{2}\right]$ & $117.76 \pm 36.19$ & $116.48 \pm 41.05$ & 0.794 & $117.68 \pm 36.26$ & $116.66 \pm 40.62$ & 0.836 \\
\hline Syntax score & $10.50(20.5)$ & $0(3)$ & $<0.001$ & $10(20.25)$ & $0(5)$ & $<0.001$ \\
\hline
\end{tabular}

MS IDF — metabolic syndrome according to International Diabetes Federation definition; MS AHA/NHLBI — metabolic syndrome according to American Heart Association/National Heart Lung Blood Institute definition; WC — waist circumference; HC — hip circumference; HDL — high density lipoprotein; LDL — low density lipoprotein; eGFR - estimated glomerular filtration rate; SBP — systolic blood pressure; DBP — diastolic blood pressure; MAP — mean arterial pressure; LVEF — left ventricular ejection fraction; LVMI — left ventricular mass index

51\% [19]. Recently, Al-Aqeedi et al. [20] reported that in ACS patients without history of diabetes MS was frequent $(69.4 \%)$ and reduced HDL increased fasting blood glucose, and triglycerides were the most frequent MS components. Although MS is frequently associated with diabetes, in the absence of diabetes MS resulted in increased cardiovascular events and mortality. The diabetes is associated with increased coronary artery lesion complexity and severity. Moreover, diabetes is generally accepted as the major underlying patholophysiological mechanism of MS causing CAD. Therefore, we excluded patients with diabetes.

Although MS was shown to predict cardiovascular events, whether MS or any of its components are independently associated with cardiovascular risk is controversial [21, 22]. MS defined according to ATP III and IDF criteria failed to predict coronary heart disease in diabetic patients $[23,24]$.
In our study, the primary aim was to evaluate the relationship between the presence of MS based on AHA/NHLBI and IDF criteria with CAD complexity assessed by SS. We also aimed at testing whether the AHA/NHLBI or IDF criteria are similarly correlated with SS. We found that SBP, DBP, age, LVEF, $\mathrm{WC} / \mathrm{HC}$ and $\mathrm{HDL}$ were the independent predictors of CAD complexity; furthermore, AHA/NHLBI and IDF criteria were similarly correlated with SS.

In the MESYAS study it was demonstrated that MS is related to a substantial increase in the risk of developing CAD [25]. MS components confer very different intensities of independent risk, from the high independent risk of hypertriglyceridemia to the almost complete absence of an independent effect of overweight (even appearing to be protective, which seems contradictory). MS is a marker of CAD risk, but not above and beyond the risk associated with its individual components. 
Table 3. The correlates of Syntax score.

\begin{tabular}{lcc}
\hline Variable & $\mathbf{r}$ & $\mathbf{p}$ \\
\hline Age & 0.157 & 0.013 \\
Sex & 0.205 & 0.001 \\
Hypertension & 0.203 & 0.001 \\
Hyperlipidemia & 0.180 & 0.005 \\
Smoking & 0.041 & 0.523 \\
Body mass index & 0.128 & 0.045 \\
Waist circumference & 0.299 & $<0.001$ \\
Hip circumference & -0.13 & 0.835 \\
Waist/Hip circumference ratio & 0.413 & $<0.001$ \\
Triglyceride & 0.071 & 0.265 \\
High density lipoprotein & -0.283 & $<0.001$ \\
Glucose & 0.177 & 0.005 \\
Low density lipoprotein & 0.006 & 0.230 \\
Total cholesterol & -0.066 & 0.299 \\
Creatinine & 0.141 & 0.026 \\
Estimated glomerular filtration rate & -0.023 & 0.719 \\
Systolic blood pressure & 0.330 & $<0.001$ \\
Diastolic blood pressure & 0.116 & 0.069 \\
Pulse pressure & 0.334 & 0.001 \\
Mean arterial pressure & 0.227 & $<0.001$ \\
MS score IDF & 0.360 & $<0.001$ \\
MS score AHA/NHLBI & 0.361 & $<0.001$ \\
MS IDF & 0.446 & $<0.001$ \\
MS AHA/NHLBI & 0.341 & $<0.001$ \\
Left ventricular ejection fraction & -0.163 & 0.010 \\
Left ventricle mass index & 0.108 & 0.091 \\
\hline IDF metaboli syndrome accoding & & \\
\hline & 0.00 \\
\hline
\end{tabular}

MS IDF - metabolic syndrome according to International Diabetes Federation definition; MS AHA/NHLBI - metabolic syndrome according to American Heart Association/National Heart Lung Blood Institute definition
It was reported that among the components of MS, abdominal obesity and low serum HDL-C were significant independent predictors for culprit coronary plaque rupture [26]. Ertek et al. [5] reported that diabetic patients had significantly higher Gensini scores among patients with MS. In this study we excluded patients with diabetes and found that patients with MS had significantly higher CAD complexity than those without implicating more bifurcation lesions, calcifications, tortuosity, extensive and diffuse involvement. These findings were compatible with the findings of Zheng et al. [16] and Marso et al. [15]. Zheng et al. [16] demonstrated that diabetic and MS patients, compared to patients without diabetes mellitus or MS, had a larger plaque-plus-media burden, larger amount of necrotic core and more frequent virtual histology intravascular ultrasound derived thin-cap fibroatheroma in coronary arterial trees, implying greater plaque vulnerability in diabetes mellitus and MS patients. Marso et al. [15] showed that patients with diabetes and MS had higher 3-year MACE rates and lesion length, plaque burden, necrotic core, and calcium content were significantly greater among nonculprit lesions of patients with diabetes and MS that presented with ACS.

\section{Limitations of the study}

The sample size is relatively small. The plasma insulin levels and insulin resistance was not studied. This was an angiographic study and as such, coronary plaque morphology was not directly

Table 4. The independent predictors of Syntax score.

\begin{tabular}{lccc}
\hline Variable & $\mathbf{p}$ & $\mathbf{B}$ & 95\% confidence interval \\
\hline Waist circumference & 0.770 & -0.40 & -0.308 to 0.228 \\
Systolic blood pressure & $<0.001$ & 0.354 & 0.224 to 0.484 \\
Diastolic blood pressure & 0.006 & -0.194 & -0.333 to -0.056 \\
Sex & 0.428 & -1.189 & -4.185 to 1.758 \\
Age & 0.014 & 0.147 & 0.029 to 0.264 \\
Body mass index & 0.157 & -0.434 & -1.038 to 0.169 \\
Creatinine & 0.819 & 0.667 & -5.073 to 6.407 \\
Hypertension & 0.248 & -2.715 & -6.403 to 0.972 \\
Hyperlipidemia & 0.345 & 1.114 & -1.237 to 3.526 \\
Left ventricular ejection fraction & 0.031 & -0.150 & -0.286 to -0.014 \\
Waist circumference/Hip circumference & $<0.001$ & 45.713 & 23.235 to 68.191 \\
Log10 glucose & 0.071 & 18.790 & -1.589 to 39.170 \\
Log10 triglyceride & 0.157 & -3.767 & -8.990 to 1.456 \\
Log10 high density lipoprotein & $<0.001$ & -22.209 & -33.298 to -11.119 \\
\hline
\end{tabular}


assessed. With quantitative coronary angiography analysis we could only measure reference diameter and percentage diameter stenosis. However, it has been reported that this measurement can be misleading, because patients with similar measurements on angiography might have different vessel and plaque volume when assessed by intravascular ultrasound which enables cross-sectional imaging of coronary arteries and provides more comprehensive assessment of atherosclerotic plaque.

\section{Conclusions}

MS is associated with the presence and complexity of CAD. Besides the presence of discrepancy about the limits of WC, both IDF and AHA/ /NHLBI criteria were similarly correlated with CAD complexity. This finding suggested that both IDF and AHA/NHLBI definitions may be used to evaluate and manage for patients at high risk for CAD.

\section{Conflict of interest: none declared}

\section{References}

1. Grundy SM, Cleeman JI, Daniels SR et al.; American Heart Association; National Heart, Lung, and Blood Institute. Diagnosis and management of the metabolic syndrome: an American Heart Association/National Heart, Lung, and Blood Institute Scientific Statement Circulation, 2005; 112: 2735-2752.

2. Alberti KG, Zimmet P, Shaw J; IDF Epidemiology Task Force Consensus Group. The metabolic syndrome: A new worldwide definition. Lancet, 2005; 366: 1059-1062.

3. Gami AS, Witt BJ, Howard DE et al. Metabolic syndrome and risk of incident cardiovascular events and death: A systematic review and meta-analysis of longitudinal studies. J Am Coll Cardiol, 2007; 49: 403-414.

4. Mottillo S, Filion KB, Genest J et al. The metabolic syndrome and cardiovascular risk. A systematic review and meta-analysis. J Am Coll Cardiol, 2010; 56: 1113-1132.

5. Ertek S, Cicero AF, Cesur M et al. The severity of coronary atherosclerosis in diabetic and non-diabetic metabolic syndrome patients diagnosed according to different criteria and undergoing elective angiography. Acta Diabetol, 2011; 48: 21-27.

6. Bayturan O, Tuzcu EM, Uno K et al. Comparison of rates of progression of coronary atherosclerosis in patients with diabetes mellitus versus those with the metabolic syndrome. Am J Cardiol, 2010; 105: 1735-1739.

7. Zornitzki T, Ayzenberg O, Gandelman G et al. Diabetes, but not the metabolic syndrome, predicts the severity and extent of coronary artery disease in women. QJM, 2007; 100: 575-581.

8. Anderson J, Horne B, Jones $\mathrm{H}$ et al. Which features of metabolic syndrome predict the prevalence and clinical outcomes of angiographic coronary artery disease? Cardiology, 2004; 101: 185-193.

9. Timóteo AT, Mota Carmo M, Cruz Ferreira R. Does metabolic syndrome predict significant angiographic coronary artery disease? Rev Port Cardiol, 2012; 31: 769-778.

10. Yoon SE, Ahn SG, Kim JY et al. Differential relationship between metabolic syndrome score and severity of coronary atherosclerosis as assessed by angiography in a non-diabetic and diabetic Korean population. J Korean Med Sci, 2011; 26: 900-905.

11. Sianos G, Morel MA, Kappetein AP et al. The SYNTAX score: an angiographic tool grading the complexity of coronary artery disease. Eurointervention, 2005; 1: 219-227.

12. Capodanno D, Di Salvo ME, Cincotta G, Miano M, Tamburino C, Tamburino C. Usefulness of the SYNTAX score for predicting clinical outcome after percutaneous coronary intervention of unprotected left main coronary artery disease. Circ Cardiovasc Interv, 2009; 2: 302-308.

13. Aykan AC, Gül I, Gökdeniz T et al. Is coronary artery disease complexity valuable in the prediction of contrast induced nephropathy besides Mehran Risk Score, in patients with ST elevation myocardial infarction treated with primary percutaneous coronary intervention? Heart Lung Circ, 2013; 22: 836-843 .

14. Gökdeniz T, Boyaci F, Hatem E et al. SYNTAX score predicts the left ventricle thrombus development in patients undergoing primary percutaneous coronary intervention for first anterior myocardial infarction. Clin Appl Thromb Hemost, Epub. 2013 Feb 22 .

15. Marso SP, Mercado N, Maehara A et al. Plaque composition and clinical outcomes in acute coronary syndrome patients with metabolic syndrome or diabetes. J Am Coll Cardiol Cardiovasc Imaging, 2012; 5 (3 suppl.): 42-52.

16. Zheng M, Choi SY, Tahk SJ et al. The relationship between volumetric plaque components and classical cardiovascular risk factors and the metabolic syndrome a 3-vessel coronary artery virtual histology-intravascular ultrasound analysis. J Am Coll Cardiol Cardiovasc Interv, 2011; 4: 503-510.

17. National Cholesterol Education Program (NCEP) Expert Panel on Detection, Evaluation, and Treatment oh High Blood Cholesterol in Adults (Adult Treatment Panel III). Third Report of the National Cholesterol Education Program (NCEP) Expert Panel on Detection, valuation, and Treatment of High Blood Cholesterol in Adults (Adult Treatment Panel III) final report. Circulation, 2002; 106: 3143-3421.

18. Alberti KG, Eckel RH, Grundy SM et al.; International Diabetes Federation Task Force on Epidemiology and Prevention; Hational Heart, Lung, and Blood Institute; American Heart Association; World Heart Federation; International Atherosclerosis Society; International Association for the Study of Obesity. Harmonizing the metabolic syndrome: A joint interim statement of the International Diabetes Federation Task Force on Epidemiology and Prevention; National Heart, Lung, and Blood Institute; American Heart Association; World Heart Federation; International Atherosclerosis Society; and International Association for the Study of Obesity. Circulation, 2009; 120: 1640-1645.

19. Malik S, Wong ND, Franklin SS et al. Impact of the metabolic syndrome on mortality from coronary heart disease, cardiovascular disease, and all causes in United States adults. Circulation, 2004; 110: 1245-1250.

20. Al-Aqeedi RF, Abdullatef WK, Dabdoob W, Bener A, Albinali HA, Gehani A. The prevalence of metabolic syndrome components, individually and in combination, in male patients admitted with acute coronary syndrome, without previous diagnosis of diabetes mellitus Libyan J Med, 2013; 8: 20185.

21. Lawlor DA, Dawey Smith G, Ebrahim S. Does the new International Diabetes Federation definition of metabolic syndrome predict $\mathrm{CHD}$ any more strongly than older definitions? Findings from the British Women's heart and health study. Diabetologia, 2006; 49: 41-48.

22. Tsai SP, Wen CP, Chan HT, Chiang PH, Tsai MK, Cheng TY. The effects of pre-disease risk factors within metabolic syndrome on all-cause and cardiovascular disease mortality. Diabetes Res Clin Pract, 2008; 82: 148-156.

23. Protopsaltis I, Nikolopoulos G, Dimou E et al. Metabolic syndrome and its components as predictors of all-cause mortality and coronary heart disease in type 2 diabetic patients. Atherosclerosis, 2007; 195: 189-194.

24. Tong PC, Kong AP, So WY et al. The usefulness of International Diabetes Federation and the National Cholesterol Education Program's Adult Treatment Panel III definitions of the metabolic syndrome in predicting coronary heart disease in subjects with type 2 diabetes. Diabetes Care, 2007; 30: 1206-1211.

25. Laclaustra M, Ordonez B, Leon M et al. Metabolic syndrome and coronary heart disease among Spanish male workers: A case-control study of MESYAS. Nutr Metab Cardiovasc Dis, 2012; 22: 510-516.

26. Kato M, Dote K, Naganuma T et al. Clinical predictors of culprit plaque rupture assessed on intravascular ultrasound in acute coronary syndromes. Circ J, 2010; 74: 1936-1942. 